\title{
Encodings of Range Maximum-Sum Segment Queries and Applications
}

\author{
Paweł Gawrychowski ${ }^{\star 1}$ and Patrick K. Nicholson ${ }^{2}$ \\ 1 Institute of Informatics, University of Warsaw, Poland \\ 2 Max-Planck-Institut für Informatik, Saarbrücken, Germany
}

\begin{abstract}
Given an array $A$ containing arbitrary (positive and negative) numbers, we consider the problem of supporting range maximumsum segment queries on $A$ : i.e., given an arbitrary range $[i, j]$, return the subrange $\left[i^{\prime}, j^{\prime}\right] \subseteq[i, j]$ such that the sum $\sum_{k=i^{\prime}}^{j^{\prime}} A[k]$ is maximized ${ }^{3}$ Chen and Chao [Disc. App. Math. 2007] presented a data structure for this problem that occupies $\Theta(n)$ words, can be constructed in $\Theta(n)$ time, and supports queries in $\Theta(1)$ time. Our first result is that if only the indices $\left[i^{\prime}, j^{\prime}\right]$ are desired (rather than the maximum sum achieved in that subrange), then it is possible to reduce the space to $\Theta(n)$ bits, regardless the numbers stored in $A$, while retaining the same construction and query time. Our second result is to improve the trivial space lower bound for any encoding data structure that supports range maximum-sum segment queries from $n$ bits to $1.89113 n-\Theta(\lg n)$, for sufficiently large values of $n$. Finally, we also provide a new application of this data structure which simplifies a previously known linear time algorithm for finding $k$-covers: given an array $A$ of $n$ numbers and a number $k$, find $k$ disjoint subranges $\left[i_{1}, j_{1}\right], \ldots,\left[i_{k}, j_{k}\right]$, such that the total sum of all the numbers in the subranges is maximized. As observed by Csürös [IEEE/ACM TCBB 2004], $k$-covers can be used to identify regions in genomes.
\end{abstract}

\section{Introduction}

Many core data structure problems involve supporting range queries on arrays of numbers: see the surveys of Navarro [16] and Skala [19] for numerous examples. Likely the most heavily studied range query problem of this kind is that of supporting range maximum queries (resp. range minimum queries): given an array $A$ of $n$ numbers, preprocess the array such that, for any range $[i, j] \subseteq[1, n]$ we can return the index $k \in[i, j]$ such that $A[k]$ is maximum (resp. minimum). These kinds of queries have a large number of applications in the area of text indexing [9, Section 3.3]. Solutions have been proposed to this problem that achieve $\Theta(n)$ space (in terms of number of machine words 4 , and constant query

* Currently holding a post-doc position at Warsaw Center of Mathematics and Computer Science.

${ }^{3}$ We use the terms segment and subrange interchangeably, but only use segment when referring to the name of the problem, for consistency with prior work.

${ }^{4}$ In this paper we assume the word-RAM model with word size $\Theta(\log n)$ bits. 
time 28]. At first glance, one may think this to be optimal, since the array $A$ itself requires $n$ words to be stored. However, if we only desire the index of the maximum element, rather than the value of the element itself, it turns out that it is possible to reduce the space [10].

By a counting argument, it is possible to show that $2 n-o(n)$ bits are necessary to answer range maximum queries on an array of $n$ numbers [10, Sec. 1.1.2]. On the other hand, rather surprisingly, it is possible to achieve this space bound, to within lower order terms, while still retaining constant query time [10. That is, regardless of the number of bits required to represent the individual numbers in $A$, we can encode a data structure in such a way as to support range maximum queries on $A$ using $2 n+o(n)$ bits. The key point is that we need not access $A$ during any part of the query algorithm. In a more broad sense, results of this type are part of the area of succinct data structures [13, in which the aim is to represent a data structure using space matching the information theoretic lower bound, to within lower order terms.

In this paper, we consider range maximum-sum segment queries [6], where, given a range $[i, j]$, the goal is to return a subrange $\left[i^{\prime}, j^{\prime}\right] \subseteq[i, j]$ such that $\sum_{k=i^{\prime}}^{j^{\prime}} A[k]$ is maximized. Note that this problem only becomes non-trivial if the array $A$ contains negative numbers. With a bit of thought it is not difficult to see that supporting range maximum queries in an array $A$ can be reduced to supporting range maximum-sum segment queries on a modified version of $A$ that we get by padding each element of $A$ with a sufficiently large negative number (see [6] for the details of the reduction). However, Chen and Chao [6] showed that a reduction holds in the other direction as well: range maximum-sum segment queries can be answered using a combination of range minimum and maximum queries on several different arrays, easily constructible from $A$. Specifically, they show that these queries can be answered in constant time with a data structure occupying $\Theta(n)$ words, that can be constructed in linear time.

A natural question one might ask is whether it is possible to improve the space of their solution to $\Theta(n)$ bits rather than $\Theta(n)$ words, while still retaining the constant query time. On one hand, we were aware of no information theoretic lower bound that ruled out the possibility of achieving $\Theta(n)$ bits. On the other hand, though Chen and Chao reduce the problem to several range maximum and range minimum queries, they still require comparisons to be made between various word-sized elements in arrays of size $\Theta(n)$ words in order to make a final determination of the answer to the query; we review the details of their solution in Section 4.1. Therefore, it was not clear by examining their solution whether further space reduction was possible.

Our first result, presented in Section 4.2, is that if we desire only the indices of the maximum-sum segment $\left[i^{\prime}, j^{\prime}\right]$ rather than the value of the sum itself, then we can achieve constant query time using a data structure occupying $\Theta(n)$ bits and constructable in linear time. There are many technical details, but the main idea is to sidestep the need for explicitly storing the numeric arrays required by Chen and Chao to make comparisons by storing two separate graphs that are judiciously defined so as to be embeddable in one page. By a well known 
theorem of Jacobson [13], combined with later improvements [15]12], it is known that one-page graphs - also known as outerplanar graphs - can be stored in a number of bits that is linear in the total number of vertices and edges, and be constructed in linear time, while still retaining the ability to navigate between nodes in constant time. Navigating these graphs allows us to implicitly simulate comparisons between certain numeric array elements, thus avoiding the need to store the arrays themselves.

Our second result, presented in Section 6 is to improve the information theoretic lower bound for this problem. It is quite trivial to show that one requires $n$ bits to support range maximum-sum segment queries by observing that if an array contains only numbers from the set $\{1,-1\}$ we can recover them via $\Theta(n)$ queries. Since there are $2^{n}$ possible arrays of this type, the lower bound follows. In contrast, by an enumeration argument, we give an improved bound of $1.89113 n-\Theta(\lg n)$ bits when $n$ is sufficiently large. The main idea is to enumerate a combinatorial object which we refer to as maximum-sum segment trees, then bound the number of trees of this type using generating functions.

Our final result, presented in Section 7, is a new application for maximumsum segment data structures. Given an array and a number $k$, we want to find a $k$-cover: i.e., $k$ disjoint subranges with the largest total sum. This problem was first studied by Csurös [7, who was motivated by an application in bioinformatics, and constructed an $\mathcal{O}(n \log n)$ time algorithm. Later, an optimal $\mathcal{O}(n)$ time solution was found by Bengtsson and Chen [4. We provide an alternative $\mathcal{O}(n)$ time solution, which is an almost immediate consequence of any constant time maximum-sum segment data structure that can be constructed in linear time. An advantage of our algorithm is that it can be also used to preprocess the array just once, and then answer the question for any $k \in[1, n]$ in $\mathcal{O}(k)$ time. We remark that this is related, but not equivalent, to finding $k$ non-overlapping maximum-sum segments, and finding $k$ maximum-sum segments. In the latter, one considers all $\left(\begin{array}{l}n \\ 2\end{array}\right)+n$ segments ordered non-increasingly according to their corresponding sums, and wants to select the $k$-th one [14]. In the former, one repeats the following operation $k$ times: find a maximum-sum segment disjoint from all the previously chosen segments, and add it to the current set [18].

\section{Notation and Definitions}

We follow the notation of Chen and Chao [6] with a few minor changes. Let $A$ be an array of $n$ numbers. Let $S(i, j)$ denote the sum of the values in the range $[i, j]$ : i.e., $S(i, j)=\sum_{k=i}^{j} A[k]$. Let $C$ be an array of length $n$ such that $C[i]$ stores the cumulative sum $S(1, i)$. Note that $S(i, j)=C[j]-C[i-1]$ if $i>1$.

Given an arbitrary array $B$, a range maximum query $\operatorname{RMAxQ}(B, i, j)$ returns the index of the rightmost maximum value in the subarray $B[i, j]$; the query $\operatorname{RMinQ}(B, i, j)$ is defined analogously. A range maximum-sum segment query RMaxSSQ $(A, i, j)$ returns a subrange $A\left[i^{\prime}, j^{\prime}\right]$ such that $i \leq i^{\prime} \leq j^{\prime} \leq j$, and $S\left(i^{\prime}, j^{\prime}\right)$ is maximum. If there is a tie, then our data structure will return the 
shortest range with the largest value of $j^{\prime}$; i.e., the rightmost one $\sqrt{5}$ Note that the answer is a range, specified by its endpoints, rather than the sum of the values in the range. Also note that if the range $A[i, j]$ contains only non-positive numbers, we return an empty range as the solution: we discuss alternatives in Appendix A but suggest reading on to Section 4 first.

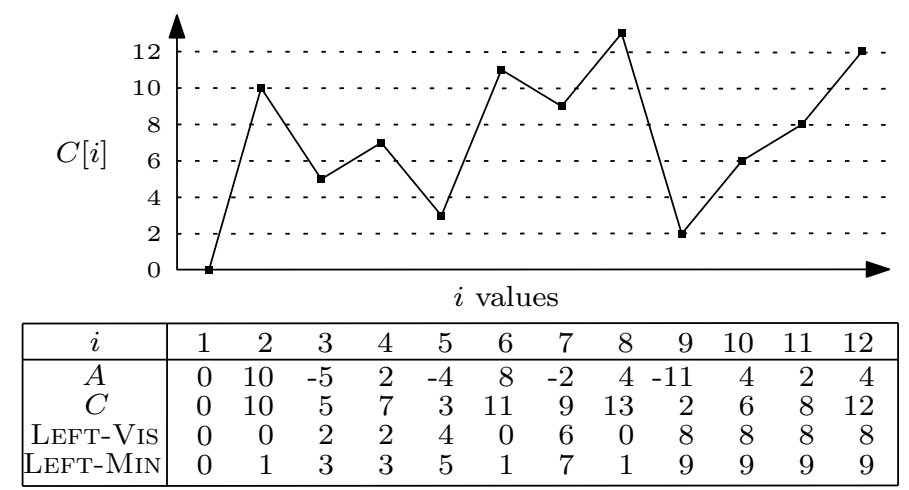

Fig. 1. Example array $A$ and the values of the various definitions presented in this section that are induced by $A$. The list of candidates for this array are: $(1,2),(3,4)$, $(1,6),(1,8),(9,10),(9,11),(9,12)$.

The left visible region $\operatorname{LEFT}-\operatorname{VIS}(i)$ of array $C$ at index $i$ is defined to be the maximum index $1 \leq j<i$ such that $C[j] \geq C[i]$, or 0 if no such index exists: this corresponds to the "left bound" definition of Chen and Chao. The left minimum $\operatorname{Left}-\operatorname{Min}(i)$ of array $C$ is defined to be $\operatorname{RMinQ}(C, \operatorname{Left}-\operatorname{Vis}(i)+1, i)$ for $1<i \leq n$. This corresponds to (one less than) the "good partner" definition of Chen and Chao. See Figure 1 for an illustration of these definitions. The pairs $(\operatorname{Left}-\operatorname{Min}(i), i)$ where $\operatorname{LEFT}-\operatorname{Min}(i)<i$ are referred to as candidates. Thus, candidates are such that the sum in $A[\operatorname{LeFT-Min}(i)+1 . . i]$ is positive. One issue is that the pair $(\operatorname{Left}-\operatorname{Min}(1), 1)$ might have a positive sum, but not be a candidate. Without loss of generality we can ignore this case by assuming $A[1]=0$, as in Figure 1. Define the candidate score array $D$ such that $D[i]=$ $S(\operatorname{Left}-\operatorname{Min}(i)+1, i)$ if $(\operatorname{Left}-\operatorname{Min}(i), i)$ is a candidate, and $D[i]=0$ otherwise, for all $i \in[1, n]$. Thus, for non-candidates, the candidate score is 0 . Let $x^{\prime}=$ $\operatorname{RMaxQ}(D, 1, n)$ and $t^{\prime}=\operatorname{Left-Min}\left(x^{\prime}\right)$. From the definitions it is not too difficult to see that $\operatorname{RMaxSSQ}(A, 1, n)$ is $\left[t^{\prime}+1, x^{\prime}\right]$ if $t^{\prime} \neq x^{\prime}$, and the empty range otherwise.

\footnotetext{
$\overline{5}$ Alternatively, we can return the leftmost such range by symmetry.
} 


\section{Preliminary Data Structures}

We make use of the following result for supporting range minimum and range maximum queries on arrays.

Lemma 1 ([10]). Given an array $B$ of $n$ numbers, we can store a data structure of size $2 n+o(n)$ bits such that $\operatorname{RMAxQ}(B, i, j)$ can be returned for any $1 \leq i \leq$ $j \leq n$ in $\mathcal{O}(1)$ time. Similarly, we can also answer $\operatorname{RMinQ}(B, i, j)$ for $1 \leq i \leq$ $j \leq n$ using the same space bound. These data structures can be constructed in linear time so as to return the rightmost maximum (resp. minimum) in the case of a tie.

We also require the following succinct data structure result for representing one-page graphs. A one-page (or outerplanar) graph $G$ has the property that it can be represented by a sequence of balanced parentheses [13]. Equivalently, there exists a labelling $1, \ldots, n$ of the vertices in $G$ in which there is no pair of edges $\left(u_{1}, u_{2}\right)$ and $\left(u_{3}, u_{4}\right)$ in $G$ such that $1 \leq u_{1}<u_{3}<u_{2}<u_{4} \leq n$. That is, if we refer to vertices by their labels, then we have that the set of ranges represented by edges are either nested or disjoint: we refer to this as the nesting property. Note that our definitions of the navigation operations differ (slightly) from the original source so as to be convenient for our application, and we explain how to support these operations in Appendix B.

Lemma 2 (Based on [15 12]). Let $G$ be a one-page multigraph with no selfloops: i.e., $G$ has vertex labels $1, \ldots, n$, and $m$ edges with the nesting property. There is a data structure that can represent $G$ using $2(n+m)+o(n+m)$ bits, and be constructed in $\Theta(n+m)$ time from the adjacency list representation of $G$, such that the following operations can be performed in constant time:

1. $\operatorname{Degree}(G, u)$ returns the degree of vertex $u$.

2. $\operatorname{Neighbour}(G, u, i)$ returns the index of the vertex which is the endpoint of the $i$-th edge incident to $u$, for $1 \leq i \leq \operatorname{DEGREE}(u)$. The edges are sorted in non-decreasing order of the indices of the endpoints: $\operatorname{NeIghBOUR}(G, u, 1) \leq$ $\operatorname{Neighbour}(G, u, 2) \leq \ldots \leq \operatorname{Neighbour}(G, u, \operatorname{Degree}(G, u))$.

3. $\operatorname{Order}(G, u, v)$ returns the order of the edge $(u, v)$ among those incident to $u$ : i.e., return an $i$ such that $\operatorname{NeIghbour}(G, u, i)=v{ }^{6}$

In all of the operations above, a vertex is referred to by its label, which is an integer in the range $[1, n]$.

\footnotetext{
${ }^{6}$ Since $G$ may be a multigraph the value of $i$ may be arbitrary among all possible values that satisfy the equation. We note that this is more general than we require, as in our application we will not execute this type of query on a multigraph, so the answer will always be unique.
} 


\section{Supporting Range Maximum-Sum Segment Queries}

In this section we present our solution to the range maximum-sum segment query problem which occupies linear space in bits. First we begin by summarizing the solution of Chen and Chao [6. Then, in Section 4.2 we describe an alternative data structure that occupies $\Theta(n)$ words of space. Finally, we reduce the space of our alternative data structure to linear in bits.

\subsection{Answering Queries using $\Theta(n)$ Words}

In the solution of Chen and Chao the following data structures are stored: the array $C$; a data structure to support $\operatorname{RMinQ}(C, i, j)$ queries; a data structure for supporting RMaxQ $(D, i, j)$ queries; and, finally, an array $P$ of length $n$ where $P[i]=\operatorname{LEFT-Min}(i)$. Thus, the overall space is linear in words.

The main idea is to examine the candidate $(P[x], x)$ whose right endpoint achieves the maximum sum in the range $[i, j]$. If $P[x]+1 \in[i, j]$ then Chen and Chao proved that $[P[x]+1, x]$ is the correct answer. However, if $P[x]+1 \notin[i, j]$ then they proved that there are two possible ranges which need to be examined to determine the answer. In this case we check the sum for both ranges and return the range with the larger sum. The pseudocode for their solution to answering the query $\operatorname{RMaxSSQ}(A, i, j)$ is presented in Algorithm 1 .

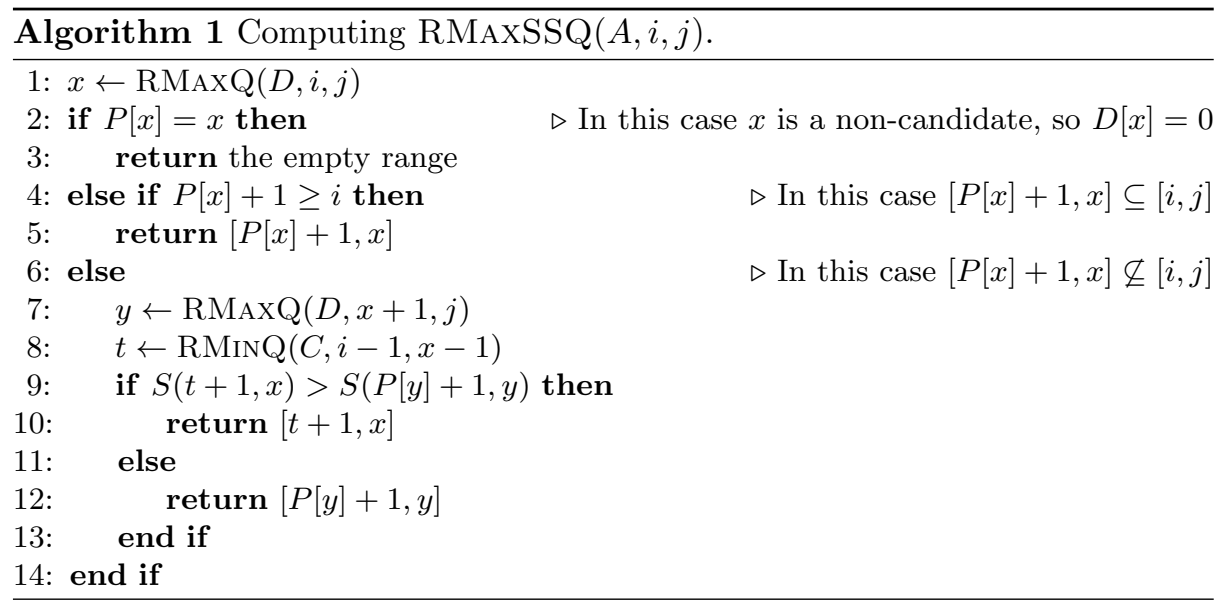

Items (1), (2) and (3) of the following collection of lemmas by Chen and Chao imply that the query algorithm is correct. We use item (4) later.

Lemma 3 ([6]). The following properties hold (using the notation from Algorithm 1):

1. If $[P[x]+1, x] \subseteq[i, j]$ then $\operatorname{RMaxSSQ}(A, i, j)$ is $[P[x]+1, x]$. 
2. The following inequalities hold: $x<P[y] \leq y$.

3. If $[P[x]+1, x] \nsubseteq[i, j]$ then $\operatorname{RMaxSSQ}(A, i, j)$ is $[P[y]+1, y]$ or $[t+1, x]$.

4. If $1 \leq i<j \leq n$ then it cannot be the case that $P[i]<P[j] \leq i$. That is, the ranges $[P[i], i]$ and $[P[j], j]$ have the nesting property for all $1 \leq i<j \leq n$.

On line 9 of Algorithm 1 the sums can be computed in constant time using the array $C$. All other steps either defer to the range maximum or minimum structures, or a constant number of array accesses. Thus, the query algorithm takes constant time to execute.

\subsection{Reducing the Space to $\Theta(n)$ Bits}

Observe that the data structure for answering RMAxQ (resp. RMinQ) queries on $D$ (resp. $C$ ) only requires $2 n+o(n)$ bits by Lemma 1 , $4 n+o(n)$ bits in total for both structures. Thus, if we can reduce the cost of the remaining data structures to $\Theta(n)$ bits, while retaining the correctness of the query algorithm, then we are done. There are two issues that must be overcome in order to reduce the overall space to linear in bits:

1. The array $P$ occupies $n$ words, so we cannot store it explicitly.

2. In the case where $[P[x]+1, x]$ is not contained in $[i, j]$, we must compare $S(t+1, x)$ and $S(P[y]+1, y)$ without explicitly storing the array $C$.

The first issue turns out to be easy to deal with: we instead encode the graph $G=([n],\{(P[x], x) \mid 1 \leq x \leq n, P[x]<x\}$, which we call the candidate graph using the following lemma:

Lemma 4. The candidate graph $G$ can be represented using $4 n+o(n)$ bits of space, such that given any $x \in[1, n]$ we can return $\operatorname{LEFT-Min}(x)$ in $\mathcal{O}(1)$ time.

Proof. Item (4) of Lemma 3 implies that the edges of $G$ have the nesting property. We store $G$ in the data structure of Lemma 2 Given $x$, we can retrieve $\operatorname{Left}-\operatorname{Min}(x)$ since, if $(\operatorname{Left}-\operatorname{Min}(x), x)$ is a candidate, we have that the degree of $x \in V(G)$ is exactly one and to a vertex with index less than $x$. If $x$ is a non-candidate, then the degree is either exactly zero, or any edge from $x$ is to a vertex with index larger than $x$, and we can return $P[x]=x$. Thus, we can navigate to $\operatorname{LeFt-Min}(x)$ in $\mathcal{O}(1)$ time.

From here onward, we can assume that we have access to the array $P$, which we simulate using Lemma 4. Unfortunately, the second issue turns out to be far more problematic. We overcome this problem via a two step approach. In the first step, we define another array $Q$ which we will use to avoid directly comparing the sums $S(t+1, x)$ and $S(P[y]+1, y)$. This eliminates the need to store the array $C$. We then show how to encode the array $Q$ using $\Theta(n)$ bits. 
Left Siblings and the $\boldsymbol{Q}$ Array: Given candidate $(P[x], x)$, we define the left sibling $\operatorname{LEFT-\operatorname {SiB}}((P[x], x))$ to be the largest index $\ell \in[1, P[x]-1]$, such that there exists an $\ell^{\prime} \in[\ell+1, P[x]]$ with $S\left(\ell+1, \ell^{\prime}\right)>S(P[x]+1, x)$, if such an index exists. Moreover, when discussing $\ell^{\prime}$ we assume that $\ell^{\prime}$ is the smallest such index. If no such index $\ell$ exists, or if $(P[x], x)$ is a non-candidate, we say $\operatorname{Left}-\operatorname{SiB}((P[x], x))$ is undefined. We define the array $Q$ such that $Q[x]=$ $\operatorname{LefT-SiB}((P[x], x))$ for all $x \in[1, n]$; if $Q[x]$ is undefined, then we store the value 0 to denote this. We now prove that we can compare $S(t+1, x)$ to $S(P[y]+1, y)$ using the $Q$ array.

Lemma 5. If $P[y]=y$ or $Q[y] \geq t$ then $\operatorname{RMaxSSQ}(A, i, j)=[t+1, x]$. Otherwise, $\operatorname{RMaxSSQ}(A, i, j)=[P[y]+1, y]$

Proof. There are several cases. For this proof, $\ell^{\prime}$ is defined relative to $Q[y]$ :

1. If $P[y]=y$ then $A[y]$ is non-positive. However, since $[P[x]+1, x] \nsubseteq[i, j]$ it is implied that $A[x]$ is positive, thus $[t+1, x]$ is the correct answer.

2. If $Q[y]>x$ then there exists an $\ell^{\prime}$ such that $S\left(Q[y]+1, \ell^{\prime}\right)>S(P[y]+1, y)$ with $\ell^{\prime}<P[y]$. Thus, $\left(Q[y], \ell^{\prime}\right)$ is a better candidate in the range $[x+1, j]$ than $(P[y], y)$, so $\operatorname{RMaxQ}(D, i+1, j)=\ell^{\prime}$ and we have a contradiction. Hence, $Q[y] \leq x$.

3. If $\left[Q[y], \ell^{\prime}\right] \subseteq[t, x]$ then by transitivity we have $S(P[y]+1, y)<S(t+1, x)$. If $Q[y] \geq t$ and $\ell^{\prime}>x$ then it implies that $S\left(x+1, \ell^{\prime}\right)$ is positive. This implies $D\left[\ell^{\prime}\right]>D[x]$, which contradicts the fact that $\operatorname{RMaxQ}(D, i, j)=x$. Thus, if $Q[y] \geq t$, then $[t+1, x]$ is the correct answer.

4. If $Q[y]<t$ then it is implied that the sum $S(t+1, x) \leq S(P[y]+1, y)$ by the definition of left sibling, so $[P[y]+1, y]$ is the rightmost correct answer. Note that if $Q[y]=0$ and $P[y] \neq y$, then $S(P[y]+1, y)$ is larger than any subrange in $[1, P[y]-1]$, so this also holds.

Note that in the previous proof, we need not know the value of $\ell^{\prime}$ in order to make the comparison: only the value $Q[y]$ is required.

Encoding the $Q$ Array: Unfortunately, the graph defined by the set of edges $(Q[x], x)$ does not have the nesting property. Instead, we construct an $n$-vertex graph $H$ using the pairs $(Q[x], P[x])$ as edges, for each $x \in[1, n]$ where $\operatorname{Left-SiB}(x)$ is defined (i.e., $Q[x] \neq 0$ ). We call $H$ the left sibling graph. We give an example illustrating both the graphs $G$ and $H$ in Figure 2. Note in the figure that each edge in $G$ has a corresponding edge in $H$ unless its left sibling is undefined. We formalize this intuition in the following lemma:

Lemma 6. Let $(P[x], x)$ be a candidate, and suppose $i=\operatorname{Degret}(H, P[x])-$ $\operatorname{Order}(G, P[x], x)+1$. If $i>0$ then it is the case that $\operatorname{Left}-\operatorname{Sib}((P[x], x))=$ $\operatorname{Neighbour}(H, P[x], i)$. Otherwise, $\operatorname{Left-\operatorname {Sib}}((P[x], x))$ is undefined.

Proof. Let $t=P[x]$ and consider the set of candidates $\left(t, x_{1}\right), \ldots,\left(t, x_{d}\right)$, where $d=\operatorname{Degree}(G, t)$, and $x_{i}=\operatorname{Neighbour}(G, t, i)$. Then, we have $S\left(t+1, x_{i-1}\right)<$ 


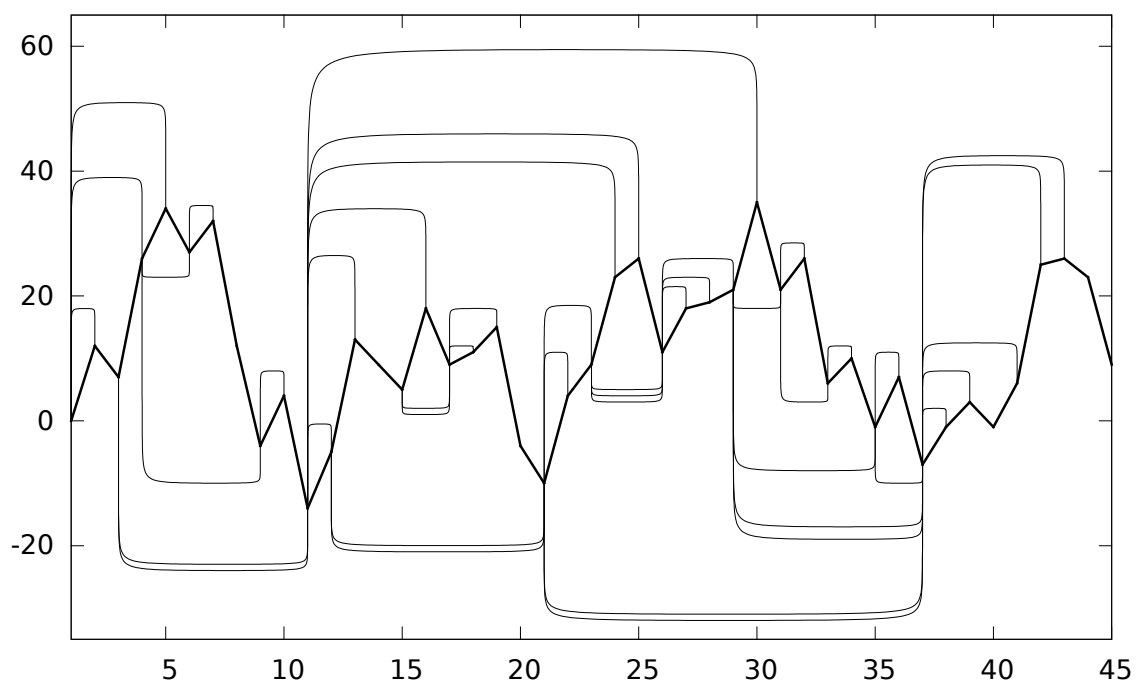

Fig. 2. A graph of the cumulative sums $C$ (thick middle line) for a randomly generated instance with $n=45$ and floating point numbers drawn uniformly from the range $[-20,20]$. The $x$-axis is the number $i$, and the $y$-axis is $C[i]$. The edges drawn above the line for $C$ represent the candidate graph $G$ and the edges below represent the left sibling graph $H$ : note that $H$ is a multigraph.

$S\left(t+1, x_{i}\right)$ for all $1<i \leq d$, since $x_{i-1}<x_{i}$ and $t$ is contained in the left visible region of all $x_{i}$. Furthermore, suppose $\ell_{j}=\operatorname{LEFT-\operatorname {SiB}}\left(\left(t, x_{j}\right)\right)$ for all $1 \leq j \leq d^{\prime}$, where $d^{\prime}=\operatorname{DegreE}(H, t)$. We show that $\ell_{j-1} \geq \ell_{j}$, by assuming the opposite. If $\ell_{j-1}<\ell_{j}$ then it is implied by the definition of left sibling that $S\left(t+1, x_{j}\right)<S\left(t+1, x_{j-1}\right)$, which is a contradiction. Thus, we have $\ell_{1} \geq \ell_{2} \geq \ldots \geq \ell_{d^{\prime}}$, and the remaining candidates $\left(t, x_{d^{\prime}+1}\right), \ldots,\left(t, x_{d}\right)$ have undefined left siblings, as there would be an edge in $H$ corresponding to them otherwise. The calculation in the statement of the lemma is equivalent to the previous statement.

Next, we prove the property that can be observed in Figure 2: namely, that we can apply Lemma 2 to $H$.

Lemma 7. The left-sibling graph $H$ can be represented using no more than $4 n+$ $o(n)$ bits of space, such that given any $x \in[1, n]$ we can return $Q[x]$ in constant time, assuming access to the data structure of Lemma 4.

Proof. If $H$ is does not have the nesting property, then there exist candidates $\left(t_{1}, x_{1}\right),\left(t_{2}, x_{2}\right)$ with $t_{1}<t_{2}$ such that $\ell_{1}<\ell_{2}<t_{1}$, where $\ell_{1}=\operatorname{LeFT-SiB}\left(\left(t_{1}, x_{1}\right)\right)$ and $\ell_{2}=\operatorname{LEFT}-\operatorname{SiB}\left(\left(t_{2}, x_{2}\right)\right)$. Proof by case analysis:

1. If $t_{2}=x_{1}$ then $\left(t_{2}, x_{2}\right)$ is not a valid candidate: the candidate with right endpoint $x_{2}$ would be $\left(t_{1}, x_{2}\right)$. 
2. If $t_{2}>x_{1}$ then we have a contradiction because $S\left(t_{2}+1, x_{2}\right)>S\left(t_{1}+1, x_{1}\right)$ (since $\ell_{2}<t_{1}$ ), and $\ell_{1}$ cannot be less than $\ell_{2}$.

3 . If $t_{2}<x_{1}$ then we have $x_{2}<x_{1}$ because candidates are nested by Lemma 3 (Item 4). However, since $\left(t_{2}, x_{1}\right)$ is not a candidate, $C\left[t_{2}\right]>C\left[t_{1}\right]$, and since $\left(t_{1}, x_{1}\right)$ is a candidate we have $C\left[x_{1}\right]>C\left[x_{2}\right]$. Thus, there must exist some $x_{3} \in\left[t_{1}, t_{2}\right]$ such that $C\left[x_{3}\right] \geq C\left[x_{2}\right]$ : otherwise $\left(t_{1}, x_{2}\right)$ would be a candidate, and not $\left(t_{2}, x_{2}\right)$. This implies $S\left(t_{1}+1, x_{3}\right)>S\left(t_{2}+1, x_{2}\right)$, and therefore we have a contradiction since $\ell_{2}$ cannot be less than $t_{1}$.

Thus, we can apply Lemma 2 to $H$, achieving the desired space bound. Using the calculation in Lemma 6 we can return $Q[x]$ in constant time for any candidate $(P[x], x)$.

Thus, to simulate the query algorithm of Chen and Chao we need: the range maximum structure for the array $D$ (Lemma 1); the range minimum structure for the array $C$ (Lemma 1); the representation of the graph $G$ (Lemma 2); the representation of the graph $H$ (Lemma 2p. We have the following theorem:

Theorem 1. There is a data structure for supporting range maximum-sum segment queries, $\operatorname{RMAxSSQ}(A, i, j)$ for any $1 \leq i \leq j \leq n$ in constant time which occupies $12 n+o(n)$ bits.

Remark 1. We note that the constant factor of 12 in Theorem 1 is suboptimal. As Rajeev Raman [17 has pointed out, the space for Lemma 4 can be reduced to $2 n+o(n)$ bits. Furthermore, we have also noted that an additional $n$ bits can be saved by combining the range maximum and minimum encodings for $D$ and $C$. However, both of these improvements are quite technical and we suspect the optimal constant factor is much lower than 9. As such, we leave determination of this optimal constant as future work.

\section{Constructing the $P$ and $Q$ Arrays}

Here we show that the arrays $P$ and $Q$ can be constructed in $\mathcal{O}(n)$ time, and therefore the construction time of the data structure from Theorem 1 is $\mathcal{O}(n)$. This follows since we can construct the adjacency list representation of the graphs $G$ and $H$ in linear time from the arrays $P$ and $Q$, respectively.

Constructing the $P$ array is rather straightforward. We only need to efficiently compute LefT-VIS $(i)$ for every $i$. This can be done using a simple stack-based procedure. Informally, we consider $i=1,2, \ldots, n$ and maintain a stack, where we keep Left-Vis $(i)$, Left-Vis(Left-Vis $(i)$ ), and so on, see Algorithm 2 More formally, we define the falling staircase of a sequence $\left(a_{1}, \ldots, a_{k}\right)$ to be the maximal sequence of indices $\left(i_{1}, \ldots, i_{s}\right)$ such that $i_{s}=k$ and $i_{j}=\max \{x$ : $x<i_{j+1}$ and $\left.a_{x} \geq a_{i_{j+1}}\right\}$ for $j=s-1, \ldots, 1$. Then LefT-Vis $(i)$ is the nextto-last element of the falling staircase of $(C[1], \ldots, C[i])$, and it is easy to see that Algorithm 2 maintain such falling staircase. Having all $\operatorname{LEFT-VIS}(i)$, we can compute all LefT-Min $(i)$ in $\mathcal{O}(n)$ total time with range minimum queries 

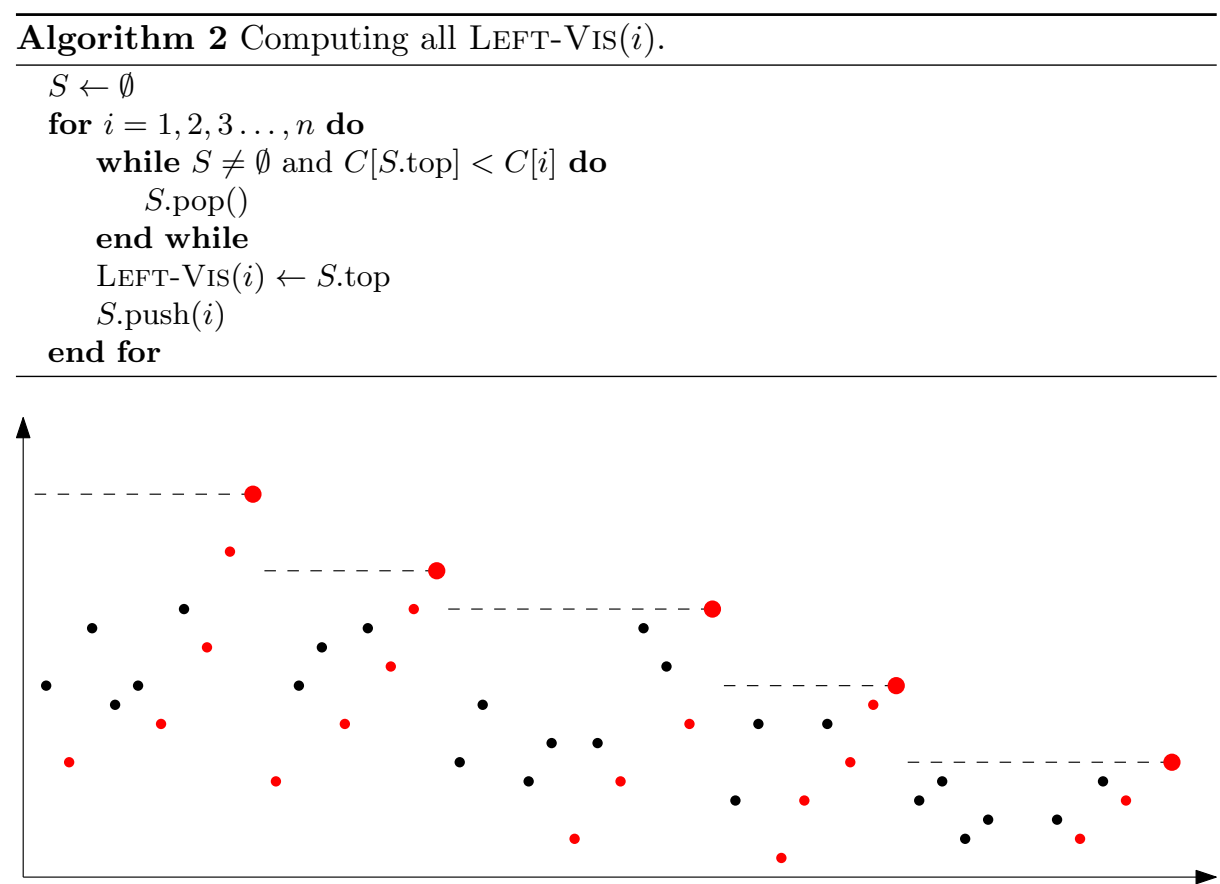

Fig. 3. A schematic depiction of the stack of rising staircases. Each rising staircase is in red, and the falling staircase consists of the larger red elements.

(by Lemma 1, a range minimum structure can be constructed in $\mathcal{O}(n)$ time and answers any query in $\mathcal{O}(1)$ time).

Computing the $Q$ array is more involved. Let $\left(i_{1}, \ldots, i_{s}\right)$ be the falling staircase of $(C[1], \ldots, C[i])$. It partitions $[1, n]$ into ranges $\left[1, i_{1}\right],\left[i_{1}+1, i_{2}\right], \ldots,\left[i_{s-1}+\right.$ $\left.1], i_{s}\right]$ For each of these ranges we store the rising staircase of the corresponding range of the $C$ array, where the rising staircase of $\left(a_{1}, \ldots, a_{k}\right)$ is the falling staircase of $\left(-a_{1}, \ldots,-a_{k}\right)$. Each of these rising staircases is stored on a stack, so we keep a stack of stacks, see Figure 3. Observe that the rising staircases allow us to extract $P[i]$ without using the range minimum structure by simply returning the leftmost element of the last rising staircase. This is because the sequence we constructed the last rising staircase for is exactly $C[(\operatorname{LEFT}-\operatorname{VIS}(i)+1]), i]$, and the leftmost element of a rising staircase is the smallest element of the sequence.

Before we argue that the rising staircases allow us to compute every $Q[i]$, we need to show that they can be maintained efficiently as we consider $i=1, \ldots, n$.

Lemma 8. The rising staircases constructed for $C[1, i]$ can be updated to be the rising staircases constructed for $C[1,(i+1)]$ in amortized $\mathcal{O}(1)$ time.

Proof. Consider the rising staircases constructed for $C[1 . . i]$, and denote the current ranges by $\left[1, i_{1}\right],\left[i_{1}+1, i_{2}\right], \ldots,\left[i_{s-1}+1, i_{s}\right]$. We first find largest $s^{\prime}$ such that $C\left[i_{s^{\prime}}\right]>C[i+1]$. Then we pop all $\left[i_{s^{\prime}}+1, i_{s^{\prime}+1}\right], \ldots,\left[i_{s-1}+1, i_{s}\right]$ from the stack 
and push $\left[i_{s^{\prime}}+1, i+1\right]$. The remaining part is to construct the rising staircase corresponding to the new range $\left[i_{s^{\prime}}+1, i+1\right]$. It can be constructed using the rising staircases corresponding to the removed ranges. More precisely, given the rising staircases of $C\left[i_{j}+1, i_{j}\right]$ and $C\left[i_{j+1}+1 . . i_{j+2}\right]$, both stored on stacks, the rising staircase of $C\left[i_{j}+1 . . i_{j+2}\right]$ can be constructed in $\mathcal{O}(1)$ amortized time. We call this merging the rising staircases. It can be implemented by popping the elements from the latter rising staircase as long as they are smaller than the first element on the former rising staircase (so the stacks should give us access to their top elements, but this is easy to add). Every popped element disappears forever, hence the whole merging procedure amortizes to $\mathcal{O}(1)$ time.

Now we are ready show how to compute $Q[i]$ given the rising staircases. Recall that $Q[i]$ is the largest $\ell<P[i]$ such that there exists $\ell^{\prime} \in[\ell, P[i]-1]$ for which $S\left(\ell+1, \ell^{\prime}\right)>S(P[i+1]+1, i+1)$. In other words, we want to find the largest $\ell<P[i]$ such that $C\left[\ell^{\prime}\right]-C[\ell]>C[i]-C[P[i]]$ for some $\ell^{\prime} \in[\ell, P[i]-1]$. Because $P[i]$ is the leftmost element of the last rising staircase. $\ell$ must belong to one of the earlier staircases. Then, because the rightmost elements on the rising staircases are nonincreasing, it is enough to consider $\ell$ and $\ell^{\prime}$ belonging to the same rising staircase, and furthermore without losing the generality $\ell^{\prime}$ is the rightmost element there. So, to summarize, we want to find the largest $\ell$ belonging to one of the rising staircases (but not the last one), such that $C\left[\ell^{\prime}\right]-C[\ell]>C[i]-C[P[i]]$, where $\ell^{\prime}$ is the rightmost element of the same rising staircase. We will first show how to determine $\ell^{\prime}$, i.e., the relevant staircase, and then the rightmost possible $\ell$ there.

We define the span of a rising staircase to be the difference between its leftmost and rightmost element (which, by definition, is the same as the difference between its smallest and largest element). We maintain the falling staircase of the sequence of spans of all rising staircases. In other words, we store the rising staircase with the largest span, then the rising staircase with the largest span on its right, and so on. By definition, the span of a rising staircase is equal to the largest possible value of $C\left[\ell^{\prime}\right]-C[\ell]$, where $\ell$ and $\ell^{\prime}$ belong to that rising staircase. Therefore, to determine $\ell^{\prime}$ we only need to retrieve the rising staircase corresponding to the next-to-last element of the falling staircase of the sequence of spans, which can be done in $\mathcal{O}(1)$ time, assuming that we can maintain that falling staircase efficiently.

Lemma 9. The falling staircase of the sequence of spans of all rising staircases can be updated in $\mathcal{O}(1)$ time after merging the two rightmost rising staircases.

Proof. Consider the two rightmost rising staircases. After merging, the rightmost element of the resulting rising staircase is the rightmost element of the latter rising staircase. The leftmost element of the resulting rising staircase is either leftmost element of the former or the latter rising staircase, depending on which one is smaller. Therefore, the largest element stays the same, and the smallest element stays the same or decreases, so the span of the new rising staircase cannot be smaller than the spans of the initial rising staircases. 
Now the falling staircase of the sequence of spans of all rising staircases can be updated by first popping its elements corresponding to the two rightmost rising staircases, and then including the span of the new rising staircase, which might require popping more elements. Because the new span is at least as large as the spans of the removed elements, this maintains the falling staircase correctly in $\mathcal{O}(1)$ amortized time.

After having determined the appropriate rising staircase, such that $\ell^{\prime}$ is the rightmost element there, we want to determine $\ell$. Denoting the rising staircase by $\left(i_{1}^{\prime}, \ldots, i_{s^{\prime}}^{\prime}\right)$, where $i_{s^{\prime}}^{\prime}=\ell^{\prime}$, we need to determine the largest $j$ such that $C\left[i_{s^{\prime}}^{\prime}\right]-C\left[i_{j}^{\prime}\right]>C[i]-C[P[i]]$. This can be done by starting with $j=s^{\prime}$ and decrementing $j$ as long as $C\left[i_{s^{\prime}}^{\prime}\right]-C\left[i_{j}^{\prime}\right] \leq C[i]-C[P[i]]$, i.e., scanning the rising staircase from right to left. A single scan might require a lot of time, but one can observe that all scanned elements can be actually removed from the rising staircase. This is because the next time we scan the same rising staircase again, the value of $C[i]-C[P[i]]$ will be at least as large as now. When the rising staircase (or more precisely its prefix) becomes a part of a longer rising staircase, the scanned elements will be outside of the surviving prefix, therefore they can be safely removed. This reduces the amortized complexity of determining a single $\ell$ to $\mathcal{O}(1)$, and gives the claimed total linear time to determine the whole $Q$ array.

\section{Lower Bound}

In this section we prove a lower bound by showing that range maximum segment sum queries can be used to construct a combinatorial object which we call maximum-sum segment trees, or MSS-trees for short. By enumerating the total number of distinct MSS-trees, we get a lower bound on the number of bits required to encode a data structure that supports range maximum segment sum queries.

\subsection{MSS-trees}

We define MSS-trees as follows. An MSS-tree for an array $A[1, n]$ is a rooted ordinal tree, i.e., a rooted tree in which the children are ordered. Each node is labelled with a range $[i, j] \subseteq[1, n]$. For technical reasons, as in the previous sections, we assume $A[1]=0$. The intuition is as follows. Suppose we execute the query $\operatorname{RMaxSSQ}(A, 1, n)$ and are given a range $[i+1, j]$. We define the drop of a query result $[i+1, j]$ to be the range $[i, j]$-i.e., a range with the left endpoint of the query result extended by one - as we find it more convenient to discuss drops rather than query results. Thus, since $A[1]=0$, all possible drops span at least two array locations, with the exception of the empty range, whose drop will be defined to be the empty range. Next, we consider the partial sums (i.e., the $C$ array), and how we can force certain drops to occur. To get a drop of $[i, j]$ we simply fix $C[i]$ to be the minimum, and $C[j]$ to be the maximum. Then, by fixing other partials sums we have (roughly) the following flexibility when setting the values of additional drops in $A$ : 
1. The drop of RMaxSSQ $(A, 1, i-1)$ can be completely arbitrary in the range $[1, i-1]$, of length at least two, or zero. Note that it is important that $A[1]=0$ to make this statement true. Furthermore, we maintain the invariant that the values in array locations $C[1], \ldots, C[i-1]$ are restricted to the range $(C[i], C[j])$, and that the minimum of these values occurs to the left of the maximum.

2. The drop of $\operatorname{RMaxSSQ}(A, j+1, n)$ can be completely arbitrary in the range $[j+1, n]$, of length at least two or zero. This follows since we know that $A[j+1]$ is a non-positive number, since $j+1$ was not contained in $[i, j]=$ $\operatorname{RMaxSSQ}(A, 1, n)$. As in the previous case, we maintain the invariant that the values $C[j+1], \ldots,[n]$ are restricted to the range $(C[i], C[j])$, and that the minimum of these values occurs to the left of the maximum.

3. The drop of RMaxSSQ $(A, i, j-1)$ can be almost completely arbitrary in the range $[i, j-1]$, with length at least two. The difference between this case and the previous is that the empty range cannot be returned as a drop, nor can a drop with left index $i+1$. This can be seen since $\operatorname{RMaxSSQ}(A, i, i+1)$ has a drop $[i, i+1]$, as $A[i+1]$ must be positive: otherwise, $A[i+1]$ would not be included as the left index of the query result for $\operatorname{RMaxSSQ}(A, 1, n)$ as it does not increase the score. Finally, we maintain the invariant that the values in array locations $C[i+1], \ldots, C[j-1]$ are restricted to the range $(C[i], C[j])$, and that the minimum of these values occurs to the left of the maximum.

The previous three situations are a bit vague about border cases, and we will clarify this in our later discussion. Because of these cases, our MSS-trees will in two flavours: either general, which will describe situations 1 and 2, or restricted, which describes situation 3. General MSS-trees may contain subtrees which are restricted, and restricted MSS-trees may contain subtrees which are general. In light of this mutual definition, we define general MSS-trees first, followed by restricted MSS-trees.

General MSS-trees Given the array $A$, and a range $\left[i_{0}, j_{0}\right]$, we construct a general tree in the following way. If $i_{0}=j_{0}$, then we return a single node labelled $\left[i_{0}, j_{0}\right]$. This is consistent with the fact that we have enforced the range to begin with a non-positive number for the general case by setting $A[1]=0$. Thus, there is only one possible type of tree when the range has length 1 . If the range $\left[i_{0}, j_{0}\right]$ is not valid (for instance if $i_{0}>j_{0}$ ), then we return an empty tree. Otherwise, we execute $\operatorname{RMaxSSQ}\left(A, i_{0}, j_{0}\right)$ and are given a drop $[i, j] \subseteq\left[i_{0}, j_{0}\right]$. We then create a node labelled with the range $[i, j]$. The node will have three children (all of which are possibly empty trees):

- The left child is a general MSS-tree constructed on the range $\left[i_{0}, i-1\right]$.

- The middle child is a restricted MSS-tree constructed on the range $[i, j-1]$.

- The right child is a general MSS-tree constructed on the range $\left[j+1, j_{0}\right]$. 
Restricted MSS-tree Given a subrange $\left[i_{0}, j_{0}\right]$, we construct a restricted MSStree as follows. If $i_{0}=j_{0}$ then we return an empty tree. If $i_{0}=j_{0}-1$, then we return a tree labelled with $\left[i_{0}, j_{0}\right]$. Otherwise, if we execute $\operatorname{RMaxSSQ}\left(A, i_{0}, j_{0}\right)$, then we will be given a drop $\left[i_{0}, j\right] \subseteq\left[i_{0}, j_{0}\right]$. This follows from the invariants since we know that $C\left[i_{0}\right]<C[k]$ for any $k \in\left[i_{0}+1, j_{0}\right]$. The root of the restricted tree is labelled with $\left[i_{0}, j\right]$, and has two children (again, possibly both empty subtrees):

1. The left child is the result of recursively constructing a restricted MSS-tree on the range $\left[i_{0}, j-1\right]$.

2. The right child is the result of recursively constructing a general MSS-tree on the range $\left[j+1, j_{0}\right]$.

\subsection{Examples}

Given any data structure that answers RMAXSSQ queries on $A$, we can construct the MSS-tree for $A$ by invoking the construction algorithm for general MSS-trees on the range $[1, n]$. As an example, we give a figure showing all possible MSS-trees for $n=3$ and $n=4$ in Figure 4 . Using the invariants described above, it is not difficult to construct arrays of lengths 3 and 4 -in which $A[1]=0$ - such each of the MSS-trees in the figure can be extracted by the procedure described above.

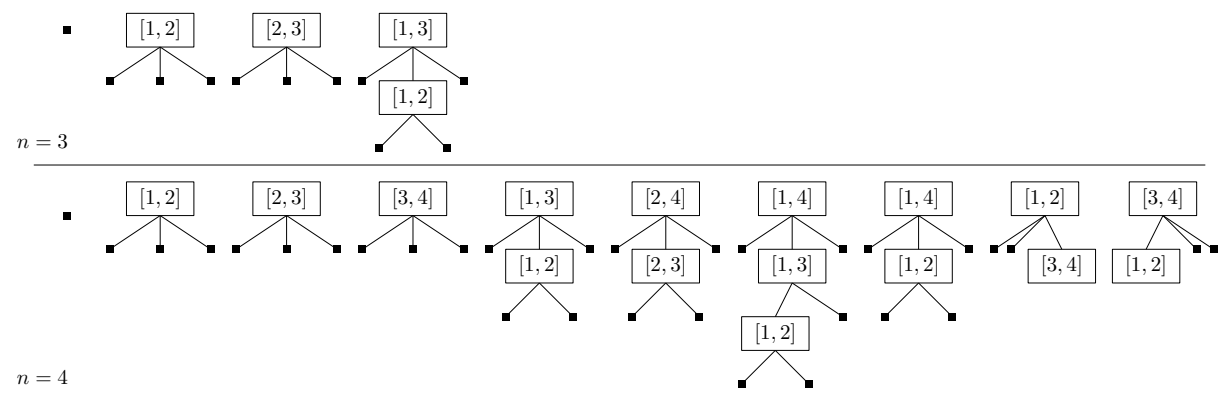

Fig. 4. Every possible MSS-tree for $n=3$ and $n=4$. Each node is a box labelled with its respective range. We use black boxes to denote empty subtrees.

\subsection{Enumeration via Recurrences}

Based on the discussion in the previous section we write the following recurrences to count the number of MSS-trees for an array of length $n$. Let $T(n)$ denote the number of general MSS-trees on an array of length $n$, and $M(n)$ denote the number of restricted trees on an array of length $n$. 


$$
\begin{aligned}
& T(0)=1 \\
& T(n)=1+\sum_{j=2}^{n} \sum_{i=1}^{j-1} T(i-1) \cdot M(j-i) \cdot T(n-j) \quad \text { for } n \geq 1 \\
& M(0)=0 \\
& M(1)=1 \\
& M(n)=\sum_{i=2}^{n} M(i-1) \cdot T(n-i) \quad \text { for } n \geq 2
\end{aligned}
$$

We rewrite (1) as follows:

$$
\begin{aligned}
T(n) & =1+\sum_{j=2}^{n} \sum_{i=1}^{j-1} T(i-1) \cdot M(j-i) \cdot T(n-j) \\
& =1+\sum_{j=2}^{n} T(n-j) \sum_{i=1}^{j-1} T(i-1) \cdot M(j-i) \\
& =1+\sum_{j=2}^{n} T(n-j) \sum_{i=1}^{j-1} M(j-i) \cdot T(i-1) \\
& =1+\sum_{j=2}^{n} T(n-j) \sum_{i=1}^{j-1} M(i) \cdot T(j-i-1) \\
& =1+\sum_{j=2}^{n} T(n-j) \sum_{i=2}^{j} M(i-1) \cdot T(j-i)
\end{aligned}
$$

Therefore, by combining with (2) we get a simpler recurrence for $T(n)$ :

$$
\begin{aligned}
T(n) & =1+\sum_{j=2}^{n} T(n-j) \cdot M(j) \\
& =1+\sum_{j=0}^{n-2} T(n-2-j) \cdot M(j+2) \quad \text { for } n \geq 1
\end{aligned}
$$

Because $M(0)=0$, we can then rewrite (2) to get the following equivalent recurrence:

$$
M(n)=\sum_{i=0}^{n-1} M(i) \cdot T(n-1-i) \quad \text { for } n \geq 2
$$

Now we define two polynomials $p(x)=\sum_{n=0}^{\infty} M(n) x^{n}$ and $q(x)=\sum_{n=0}^{\infty} T(n) x^{n}$. From (3) and (4) we get the following equalities.

$$
p(x)=x+x p(x) q(x)
$$




$$
q(x)=\frac{1}{1-x}+x^{2} q(x) \frac{p(x)-x}{x^{2}}
$$

After substituting $p(x)=\frac{x}{1-x q(x)}$ we get:

$$
\begin{aligned}
q(x) & =\frac{1}{1-x}+x^{2} q(x) \frac{p(x)-x}{x^{2}} \\
& =\frac{1}{1-x}+q(x)\left(\frac{x}{1-x q(x)}-x\right) \\
& =\frac{1}{1-x}+\frac{x^{2} q^{2}(x)}{1-x q(x)}
\end{aligned}
$$

and then:

$$
\begin{aligned}
q(x)(1-x)(1-x q(x)) & =(1-x q(x))+x^{2} q^{2}(x)(1-x) \\
q(x)-x q^{2}(x)-x q(x)+x^{2} q^{2}(x) & =1-x q(x)+x^{2} q^{2}(x)(1-x) \\
q(x)-x q^{2}(x) & =1-x^{3} q^{2}(x) \\
q^{2}(x)\left(x^{3}-x\right)+q(x)-1 & =0
\end{aligned}
$$

So finally $q(x)=\frac{1 \pm \sqrt{1-4 x\left(1-x^{2}\right)}}{2 x\left(1-x^{2}\right)}$. We can eliminate the positive branch through a simple sanity check by setting $x=0$. Thus, the generating function for the above sequence of numbers is:

$$
q(x)=\frac{1-\sqrt{1-4 x\left(1-x^{2}\right)}}{2 x\left(1-x^{2}\right)} .
$$

Interestingly, this generating function implies that the number of valid MSStrees for $n=0,1,2, \ldots$ corresponds to OEIS A157003 1 . As for the asymptotics: the first singularity encountered along the positive real axis for the function $q(x)$ is located at $x \approx 0.2695944$. By Pringsheim's Theorem [11, see p.226 and Theorems IV.6, IV.7] this implies that at least $\log _{2}\left(\frac{1}{0.269594^{n} \text { poly (n) }}\right) \geq 1.89113 n-$ $\Theta(\lg n)$ bits are required to represent an MSS-tree, provided $n$ is sufficiently large. Thus, we have proven the following theorem:

Theorem 2. For an array $A$ of length $n$, any data structure that encodes the solution to range maximum-sum segment queries must occupy at least $1.89113 n-$ $\Theta(\lg n)$ bits, if $n$ is sufficiently large.

\section{Application to Computing $k$-Covers}

Given an array $A$ of $n$ numbers and a number $k$, we want to find $k$ disjoint segments $\left[i_{1}, j_{1}\right], \ldots,\left[i_{k}, j_{k}\right]$, called a $k$-cover, such that the total sum of all numbers inside, called the score, is maximized. For $k=1$ (the RMAXSSQ problem 
on the entire array) this is a classic exercise, often used to introduce dynamic programming. For larger values of $k$, it is easy to design an $\mathcal{O}(n k)$ time dynamic programming algorithm, but an interesting question is whether we can do better. As shown by Csurös [7, one can achieve $\mathcal{O}(n \log n)$ time complexity. This was later improved to $\mathcal{O}(n \alpha(n, n))$ [3] and finally to optimal $\mathcal{O}(n)$ time [4]. In this section we show that, assuming a constant time range maximum-sum segment structure, which can be constructed in linear time, we can preprocess the array in time $\mathcal{O}(n)$, so that given any $k$, we can compute a maximum $k$-cover in $\mathcal{O}(k)$ time. This improves the previous linear time algorithm, which needs $\mathcal{O}(n)$ time to compute a maximum $k$-cover regardless of how small $k$ is, so our algorithm is more useful when there are multiple different values of $k$ for which we want to compute a maximum $k$-cover.

We iteratively construct a maximum score $k$-cover for $k=0,1,2, \ldots, n$. This is possible due to the following property already observed by Csurös.

Lemma 10. A maximum score $(k+1)$-cover can be constructed from any maximum score $k$-cover consisting of intervals $\left[i_{1}, j_{1}\right], \ldots,\left[i_{k}, j_{k}\right]$ in one of the two ways:

1. adding a new interval $\left[i_{k+1}, j_{k+1}\right]$ disjoint with all $\left[i_{1}, j_{1}\right], \ldots,\left[i_{k}, j_{k}\right]$,

2. replacing some $\left[i_{\ell}, j_{\ell}\right]$ with two intervals $\left[i_{\ell}, j^{\prime}\right],\left[i^{\prime}, j_{\ell}\right]$.

As any such transformation results in a valid $(k+1)$-cover, we can construct a maximum score $(k+1)$-cover by simply choosing the one increasing the score the most. In other words, we can iteratively select the best transformation. Now the question is how to do so efficiently.

We will first show that the best transformation of each type can be found in $\mathcal{O}(1+k)$ time using the range maximum-sum queries. Assume that we have both a range maximum-sum and a range minimum-sum query structure available. Recall that out of all possible transformations of every type, we want the find the one increasing the score the most.

1. To add a new interval $\left[i_{k+1}, j_{k+1}\right]$ disjoint with all $\left[i_{1}, j_{1}\right], \ldots,\left[i_{k}, j_{k}\right]$ increasing the score the most, we guess an index $\ell$ such that the new interval is between $\left[i_{\ell}, j_{\ell}\right]$ and $\left[i_{\ell+1}, j_{\ell+1}\right]$ (if $\ell=0$ we ignore the former and if $\ell=k$ the latter condition). Then $\left[i_{k+1}, j_{k+1}\right]$ can be found with $\operatorname{RMaxSSQ}\left(A, i_{\ell}+1, j_{\ell+1}-1\right)$.

2. To replace some $\left[i_{\ell}, j_{\ell}\right]$ with two intervals $\left[i_{\ell}, j^{\prime}\right],\left[i^{\prime}, j_{\ell}\right]$ increasing the score the most, we observe that the score increases by $-S\left(j^{\prime}+1, i^{\prime}-1\right)$, hence we can guess $\ell$ and then find $\left(j^{\prime}+1, i^{\prime}-1\right)$ with $\operatorname{RMinSSQ}\left(A, i_{\ell}, j_{\ell}\right)$.

For every type, we need $1+k$ calls to one of the structures. If each call takes constant time, the claimed $\mathcal{O}(1+k)$ complexity follows.

We will now show that, because we repeatedly apply the best transformation, the situation is more structured and the best transformation of each type can be found faster. To this end we define a transformation tree as follows. Its root corresponds to the maximum-sum segment $[i, j]$ of the whole $A$, meaning that 
its weight is $S(i, j)$, and has up to three children. If $A$ is empty or consists of only negative numbers, the transformation tree is empty.

1. The left child is the transformation tree recursively defined for $A[1 . . i-1]$.

2. The middle child is the transformation tree recursively defined for $-A[i . . j]$, i.e., for a copy of $A[i . . j]$ with all the numbers multiplied by -1 .

3. The right child is the transformation tree recursively defined for $A[j+1 . . n]$.

If any of these ranges is empty, we don't create the corresponding child. Now the transformation tree is closely related to the maximum score $k$-covers.

Lemma 11. For any $k \geq 1$, a $k$-cover constructed by the iterative method corresponds to a subtree of the transformation tree containing the root.

Proof. We apply induction on $k$. For $k=1$, the cover is exactly the maximumsum segment, which corresponds to the root of the whole transformation tree. Now assume that the lemma holds for some $k \geq 1$ and consider how the iterative method proceeds. It is easy to see that any of the possible transformations, i.e., either adding a new interval or splitting an existing interval into two, correspond to a child of a node already in the subtree corresponding to the maximum $k$ cover by the inductive hypothesis. Hence the lemma holds for $k+1$ and so for all $k \geq 1$.

This suggests that a maximum $k$-cover can be found by computing a maximum weight subtree of the transformation tree containing the root and consisting of $k$ nodes. Indeed, any such subtree corresponds to a $k$-cover, and by Lemma 11 a maximum $k$-cover corresponds to some subtree. To find a maximum weight subtree efficiently, we observe the following property of the transformation tree.

Lemma 12. The transformation tree has the max-heap property, meaning that the weight of every node is at least as large as the weight of its parent.

Proof. We apply induction on $n$. For the induction step, we need to prove that the weight of the root is at least as large as the weight of all of its children. This is immediate in case of the left and the right child, because the weight of the root is the largest $S(i, j)$ for $1 \leq i \leq j \leq n$, the weight of the left child is the largest $S\left(i^{\prime}, j^{\prime}\right)$ for $1 \leq i^{\prime} \leq j^{\prime}<i$, and the weight of the right child is the largest $S\left(i^{\prime}, j^{\prime}\right)$ for $j<i^{\prime} \leq j^{\prime} \leq n$. The weight of the middle child, if any, is the largest $-S\left(i^{\prime}, j^{\prime}\right)$ for $i \leq i^{\prime} \leq j^{\prime} \leq j$, and to finish the proof we need to argue that any such $-S\left(i^{\prime}, j^{\prime}\right)$ is at most $S(i, j)$. But if $-S\left(i^{\prime}, j^{\prime}\right)>S(i, j)$, then $\frac{S\left(i, i^{\prime}-1\right)+S\left(j^{\prime}+1, j\right)}{2}>S(i, j)$, so either $S\left(i, i^{\prime}-1\right)>S(i, j)$ or $S\left(j^{\prime}+1, j\right)>S(i, j)$. In either case, $S(i, j)$ was not a maximum-sum segment, a contradiction.

Therefore, to find a maximum weight subtree consisting of $k$ nodes, we can simply choose the $k$ nodes with the largest weight in the whole tree (we assume that the weights are pairwise distinct, and if not we break the ties by considering the nodes closer to the root first). This can be done by first explicitly constructing the transformation tree, which takes $\mathcal{O}(n)$ time assuming a constant time 
maximum and minimum range-sum segment structures. Then we can use the linear time selection algorithm [5] to find its $k$ nodes with the largest weight. This is enough to solve the problem for a single value of $k$ in $\mathcal{O}(n)$ time.

If we are given multiple values of $k$, we can process each of them in $\mathcal{O}(k)$ time assuming the following linear time and space preprocessing. For every $i=$ $0,1,2, \ldots, \log n$ we select and store the $2^{i}$ nodes of the transformation tree with the largest weight. This takes $\mathcal{O}(n+n / 2+n / 4+\ldots)=\mathcal{O}(n)$ total time and space. Then, given $k$, we find $i$ such that $2^{i} \leq k<2^{i+1}$ and again use the linear time selection algorithm to choose the $k$ nodes with the largest weight out of the stored $2^{i+1}$ nodes.

\section{References}

1. OEIS Foundation Inc. (2011), The On-Line Encyclopedia of Integer Sequences, Transform of Catalan numbers whose Hankel transform gives the Somos-4 sequence. http://oeis.org/A157003, accessed: 2014-09-24

2. Bender, M.A., Farach-Colton, M.: The LCA problem revisited. In: Proc. LATIN. LNCS, vol. 1776, pp. 88-94. Springer (2000)

3. Bengtsson, F., Chen, J.: Computing maximum-scoring segments in almost linear time. In: Proc. COCOON. pp. 255-264. LNCS, Springer, Berlin, Heidelberg (2006)

4. Bengtsson, F., Chen, J.: Computing maximum-scoring segments optimally. Tech. rep., Research Report, Luleå University of Technology (2007)

5. Blum, M., Floyd, R., Pratt, V., Rivest, R., Tarjan, R.: Time bounds for selection. Journal of Computer and System Sciences 7, 448-461 (1972)

6. Chen, K.Y., Chao, K.M.: On the range maximum-sum segment query problem. Discrete Applied Mathematics 155(16), 2043-2052 (2007)

7. Csürös, M.: Maximum-scoring segment sets. IEEE/ACM Trans. Comput. Biology Bioinform. 1(4), 139-150 (2004)

8. Durocher, S.: A simple linear-space data structure for constant-time range minimum query. In: Space-Efficient Data Structures, Streams, and Algorithms - Papers in Honor of J. Ian Munro on the Occasion of His 66th Birthday. LNCS, vol. 8066, pp. 48-60. Springer (2013)

9. Fischer, J.: Data Structures for Efficient String Algorithms. Ph.D. thesis, LudwigMaximilians-Universität München (October 2007)

10. Fischer, J., Heun, V.: Space-efficient preprocessing schemes for range minimum queries on static arrays. SIAM J. Comput. 40(2), 465-492 (2011)

11. Flajolet, P., Sedgewick, R.: Analytic Combinatorics. Cambridge Uni. Press (2009)

12. Geary, R.F., Rahman, N., Raman, R., Raman, V.: A simple optimal representation for balanced parentheses. Theor. Comput. Sci. 368(3), 231-246 (2006)

13. Jacobson, G.: Space-efficient static trees and graphs. In: Proceedings of the 30th Annual Symposium on Foundations of Computer Science. pp. 549-554. SFCS '89, IEEE Computer Society, Washington, DC, USA (1989)

14. Liu, H.F., Chao, K.M.: Algorithms for finding the weight-constrained $k$ longest paths in a tree and the length-constrained $k$ maximum-sum segments of a sequence. Theor. Comput. Sci. 407(1-3), 349-358 (Nov 2008)

15. Munro, J.I., Raman, V.: Succinct representation of balanced parentheses and static trees. SIAM J. Comput. 31(3), 762-776 (2001)

16. Navarro, G.: Spaces, trees, and colors: The algorithmic landscape of document retrieval on sequences. ACM Comput. Surv. 46(4), 52 (2013) 
17. Raman, R.: Personal communication.

18. Ruzzo, W.L., Tompa, M.: A linear time algorithm for finding all maximal scoring subsequences. In: Proceedings of the Seventh International Conference on Intelligent Systems for Molecular Biology. pp. 234-241. AAAI Press (1999)

19. Skala, M.: Array range queries. In: Space-Efficient Data Structures, Streams, and Algorithms - Papers in Honor of J. Ian Munro on the Occasion of His 66th Birthday. LNCS, vol. 8066, pp. 333-350. Springer (2013) 


\section{A Alternatives to the Empty Range}

Although the data structure we describe returns the empty range in the case that $[i, j]$ only contains non-positive numbers, it is a simple modification of our data structure to return the index of the largest non-positive number instead. To do this, we keep an additional data structure that supports range maximum queries on $A$ : this occupies $2 n+o(n)$ bits by Lemma 1 . Whenever our data structure returns the empty range, we can instead return the result of $\operatorname{RMAxQ}(A, i, j)$ query, which has the desired effect.

\section{B Navigation in One-Page Graphs}

We give a description of Munro and Raman's representation, with slight modification [15]. Given a one-page graph $G$ there is an implicit labelling of the vertices from left-to-right along its book spine. We represent such a graph using a sequence of balanced parenthesis sequence $B$. Each vertex $u$ is represented as a pair "()", and the edges incident to the vertex are represented either as an opening or closing parenthesis sequence $S_{u}$ that follow this pair. For the purposes of exposition we orient the edges so that edge $(u, v)$, where $u<v$ is directed from $u$ to $v$ : this is just to simpify the description, the edges are actually undirected. Consider the vertex labelled $u \in[1, n]$. Each edge directed into $u$ is represented in the prefix of $S_{u}$, and each edge directed out of $u$ is represented in the suffix. Let $v_{z}=\operatorname{Neighbour}(u, z)$. Then $\left(v_{1}, u\right),\left(v_{2}, u\right), \ldots,\left(v_{x}, u\right)$ are the edges directed into $u$, and $\left(u, v_{x+1}\right), \ldots,\left(u, v_{x+y}\right)$ are the edges directed out of $u$, for some $x+y=\operatorname{DegreE}(u)$. Then the sequence $\left.S_{u}=\right)^{x}\left({ }^{y}\right.$. The $i$-th ")" from left-to-right represenents the edge $\left(v_{x-i+1}, u\right)$, whereas the $i$-th "( "from left-to-right represents the edge $\left(u, v_{x+i}\right)$.

We construct the data structure of Geary et al. [12 on $B$. We use this particular structure, since has a simple construction algorithm that takes $\mathcal{O}(n+m)$ deterministic worst-case time (actually it can be constructed in $o(n+m)$ time, but we only need the weaker fact). The data structure for $B$ occupies $2(n+m)+$ $o(n+m)$ bits: note that $m$ is not necessarily $\mathcal{O}(n)$ in general since, although the graph $G$ is planar, it is a multigraph.

We also build rank/select auxiliary structures $W_{1}$ on the balanced parenthesis sequence $B$, each "()" will represent a 1 bit and all other combinations of pairs of parenthesis represent a 0 . This takes $o(n+m)$ bits in total, since we need not store the bit vector explicitly, just lookup tables [15: the details here are rather technical, but mainly involve specialized table lookup. This allows us, given a label $u$, to jump immediately to the balanced parenthesis pair "()" that represents vertex $u$ using a select operation on $W_{1}$. Similarly, given an arbitrary open/close parenthesis at position $i$, we can use the rank operation on $W_{1}$ to compute the vertex associated with that open/close parenthesis. Given this representation, it is easy to compute $\operatorname{DeGREE}(u)$ of some vertex $u$ : we simply return the distance (minus one) between the ending ")" of the pair representing $u$, and the starting "(" representing $u+1$. 
The representation just described almost allows us to perform the operation $\operatorname{Neighbour}(G, u, i)$. The issue is that listing the neighbours in the obvious way returns them in a slightly strange order: we get the edges directed into $u$ in non-increasing order of their starting vertex, followed by the edges directed out of $u$ in non-decreasing order of their starting vertex. In fact, for our application this is enough, because in our application all of the vertices either have no edges directed out, or no edges directed in. Thus, we can use $\operatorname{DegReE}(u)$, and rank/select operations on $W_{1}$ to return the neighbours in non-decreasing order. The $\operatorname{Order}(G, u, v)$ operation becomes trivial as well, since, it is known that if $(u, v)$ is an edge, we can find the pair of parentheses that represent $(u, v)$ in constant time using the representation just described. Once we have either the opening or closing parenthesis representing $(u, v)$ in $S_{u}$, we can easily compute its order using DeGreE $(u)$ and rank/select operations on $W_{1}$.

However, in the more general setting described in the lemma it is not difficult to perform all these operations as described. In addition to the previous data structures, we construct auxiliary structures $W_{2}$ which mark, for each vertex $u$, the point in $S_{u}$ where the first opening parenthesis appears. The $W_{2}$ structures allow us to perform rank and select operations on these marked positions. These also occupy $o(n+m)$ bits if used in combination with $W_{1}$. The details are, again, rather technical, but the idea is to used specialized lookup tables, bit masking, and auxiliary rank/select structures. The purpose of $W_{2}$ is to allow us to perform the operations Order and Neighbour, as the closing parenthesis in the prefix of $S_{u}$ are stored in non-ascending order of their endpoints. It is not difficult to see that using rank and select on $W_{2}$, together with a small calculation, allow us to support Order and NeIghbour operations in constant time.

As for construction time, given the adjacency list representation, we can determine for all vertices $u \in V(G)$ the string $S_{u}$ in $\mathcal{O}(n+m)$ time in total. This allows us to write down the balanced parenthesis sequence in $\mathcal{O}(n+m)$ time. 